\title{
Dissecting the region around IceCube-170922A: the blazar TXS 0506+056 as the first cosmic neutrino source
}

\author{
Theo Glauch ${ }^{1,4, *}$, Paolo Padovani ${ }^{2}$, Paolo Giommi ${ }^{3,4,5}$, Elisa Resconi ${ }^{1}$, Bruno Arsioli ${ }^{6,7}$, \\ Narek Sahakyan ${ }^{8}$, and Matthias Huber ${ }^{1}$ \\ ${ }^{1}$ Technische Universität München, Physik-Department, James-Frank-Str.1, D-85748 Garching bei \\ München, Germany \\ ${ }^{2}$ European Southern Observatory, Karl-Schwarzschild-Str. 2, D-85748 Garching bei München, Ger- \\ many \\ ${ }^{3}$ Agenzia Spaziale Italiana, ASI, via del Politecnico s.n.c., I-00133 Roma Italy \\ ${ }^{4}$ Institute for Advanced Studies, Technische Universität München, Lichtenbergstrasse 2a, D-85748 \\ Garching bei München, Germany \\ ${ }^{5}$ ICRANet, Piazzale della Repubblica, 10 - 65122, Pescara, Italy \\ ${ }^{6}$ Instituto de Física Gleb Wataghin, UNICAMP, R. Sérgio Buarque de Holanda 777 \\ ${ }^{7}$ ICRANet-Rio, CBPF, Rua Dr. Xavier Sigaud 150, 22290-180 URCA, Rio de Janeiro, Brazil \\ ${ }^{8}$ ICRANet-Armenia, Marshall Baghramian Avenue 24a, 0019 Yerevan, Republic of Armenia
}

\begin{abstract}
On MJD 58018 the IceCube neutrino observatory detected a highlyenergetic, well-reconstructed neutrino, IceCube-170922A, at a distance of $0.1^{\circ}$ to a $\gamma$-ray flaring blazar, TXS $0506+056$. Follow-up searches in archival data additionally revealed a larger flare of neutrinos from the same direction. In order to complete the picture we present here a full multi-wavelength study of the region around IceCube-170922A. While we identify also other non-thermal counterpart candidates, we show that all the evidence points to TXS $0506+056$ as the dominant neutrino emitter. Additionally, an analysis of all the available Fermi-LAT data indicates a hard spectrum/low flux of TXS 0506+056 during the neutrino flare in contrast to a soft spectrum/high flux at the arrival time of IceCube-170922A. Putting all the pieces together we conclude that the SED of TXS 0506+056 can be energetically reconnected for both neutrino observations.
\end{abstract}

\section{Introduction}

The IceCube Neutrino Observatory has recently found evidence for an astrophysical neutrino flux above $\sim 100 \mathrm{TeV}$ [1]. The detection of these neutrinos mainly happens in two channels: starting events and through-going tracks. Despite many efforts the origin of these neutrinos is still unknown [2]. Complementary to the usual point-search searches, IceCube has therefore constructed a realtime alert system [3]. It is triggered by the highest-energy tracks with very good angular resolution and sends an alert to the astronomical community to allow for time simultaneous follow-ups.

On MJD 58018 a highly-energetic, up-going muon-track, IceCube-170922A, passed the realtime event selection and was send out as an alert through the Gamma-ray Coordinates

\footnotetext{
*e-mail: theo.glauch@tum.de
} 
Network. It has a most probable neutrino energy of $\sim 290 \mathrm{TeV}$ and a best-fit direction of right ascension $77.34_{-0.65}^{\circ+0.95}$ and declination $+5.72_{-0.30}^{\circ+0.50}$. Most notably, a radio bright and flaring $\gamma$ ray blazar, TXS $0506+056$ (IBL/HBL at $\mathrm{z}=0.3365$ [4]), was found at a distance of $0.1^{\circ}$. The post-trial chance of such a coincidence is $3-3.5 \sigma$ [5]. Searches in archival IceCube data additionally revealed a flare of neutrinos during a 5-month period in 2014-2015 ${ }^{1}$ spatially consistent with the neutrino alert. The fluence of this flare is $2.1_{-0.7}^{+0.9} \times 10^{-4} \mathrm{TeV} \mathrm{cm}^{-2}$, which results in a post-trial significance of $3.5 \sigma$ [6]. Due to the absence of emission lines TXS $0506+056$ was originally classified as BL Lac. Recent studies suggest, however, that it is intrinsically an FSRQ with hidden broad line regions [8].

Despite TXS 0506+056 we identified another known $\gamma$-ray source, PKS 0502+049, as well as five non-thermal objects in the vicinity of IceCube-170922A. Based on their radio to X-ray emission ratio a prior all of them are also good counterpart candidates. Hence, in order to complete the observational picture we present here what we call a dissection of the region around IceCube-170922A. All available astronomical data are collected and analyzed in order to identify and characterize the neutrino's multi-wavelength counterpart(s) over the entire energy spectrum.

\section{Multi-Messenger Data Analysis}

Most important for the identification of the multi-wavelength counterpart candidates, data of several telescopes have been analyzed using the VOU-Blazar Tool that has been developed in the framework of the "Open Universe" 2 . The tool selects sources that emit in both, radio and X-ray with a blazar like emission ratio, as determined in the BZCAT [9]. Input Radio and optical data are taken from the Owens Valley Radio Observatory (OVRO7), the Catalina Real time Transient Survey (CRTS8) and the All Sky Automatic Survey (ASAS9) online services. The largest fraction of the X-ray data in a radius of 80 arc-minutes around IceCube-170922A comes from observations of the Neil Gehrels Swift observatory and the NuSTAR hard X-ray observatory. The preparation of all the data has been done using the standard pipelines of the respective telescopes.

In Figure 1 the region around IceCube-170922A is shown with the corresponding 90\% error contour and all the identified counterpart candidates. Despite the two $\gamma$-ray blazars (source 1 and 5), there is another known HBL, 2WHSP J050833.3+05310 (source 3), as well as an HBL candidate (source 4). Visual inspection of the remaining SEDs revealed that source 7 is a cluster of galaxies, source 6 is a steep-radio object and source 2 is a nearby elliptical galaxy. These sources are in general not capable of accelerating protons to very high energies or provide enough target material for neutrino production, hence we can exclude them.

\section{$2.1 \gamma$-ray Analysis}

The production spectra of neutrinos and $\gamma$-rays in astrophysical sources are closely connected over the process of pion decay. Hence, in the following, we study the connection of the two messengers for the remaining counterpart candidates. The presented analysis is based on 9.5 years of Fermi-LAT data and uses the standard pipelines implemented in the Fermi Science Tools. The sensitive energy range of Fermi-LAT starts at $100 \mathrm{MeV}$ and ends at $100 \mathrm{GeV}$ - exactly where the energy range of IceCube begins. Hence we are naturally interested in

\footnotetext{
${ }^{1}$ Using two different parameterizations of the flare's shape the time window is evaluated to MJD 56939 to MJD 57096 (158 days) for a box-shape and MJD 56949 to MJD 57059 ( $110_{-24}^{+35}$ days) for a Gaussian shape, respectively [6]. Both approaches are consistent within errors. While the 110 days period was extensively discussed in [7], we will present in this proceeding the analogous results for the 158 day period.

${ }^{2} \mathrm{http}: / /$ www.openuniverse.asi.it/
} 

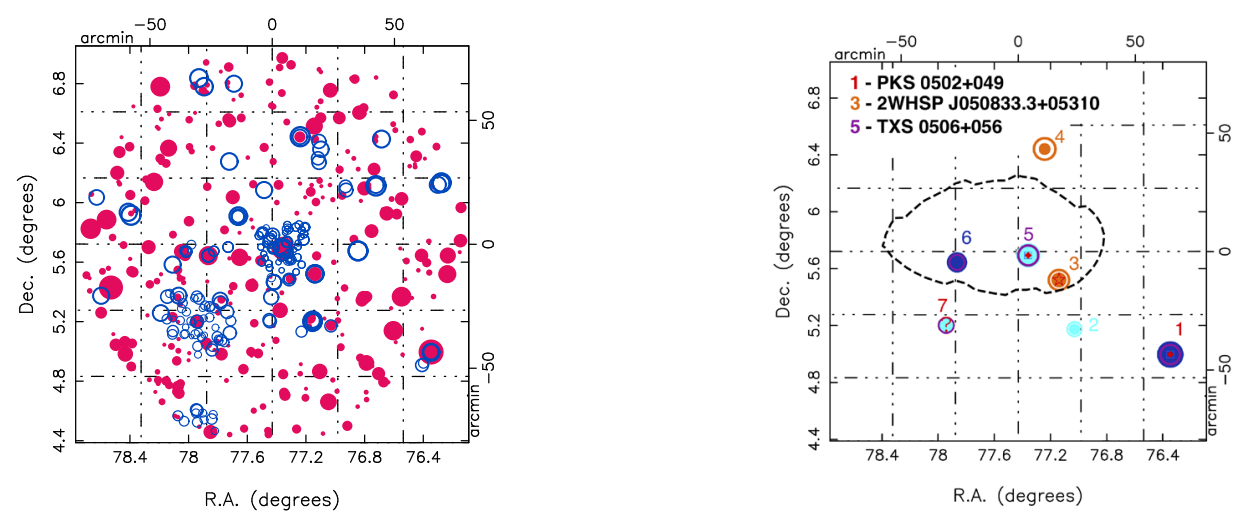

Figure 1. Left: Radio and X-ray sources within 80 arc-minutes of the position of IceCube-170922A. Symbol diameters are proportional to source intensity. Radio sources appear as red filled circles, X-ray sources as open blue circles. Right: Known and candidate blazars around IceCube-170922A. Dark blue circles represent LBL type candidates, cyan symbols are for IBL candidates, and orange symbols are for HBL candidates. Known blazars are marked by a red diamond if they are included in the BZCAT catalogue or a star if they are part of the 2WHSP sample. The dashed line shows the $90 \%$ error contour of IceCube-170922A.

the high-energy part of the Fermi-LAT range. Additionally, in order to avoid any source confusion due to wrongly modeled sources, we mostly exclude photon energies below $2 \mathrm{GeV}$ (see figure captions and [7] for more details). The test-statistic (TS) maps in Figure 2 show the $\gamma$-ray emission for the time windows around the neutrino alert and during the neutrino flare (significance $\sigma \sim \sqrt{T S}$ ). It can be seen that there is no obvious emission from the direction
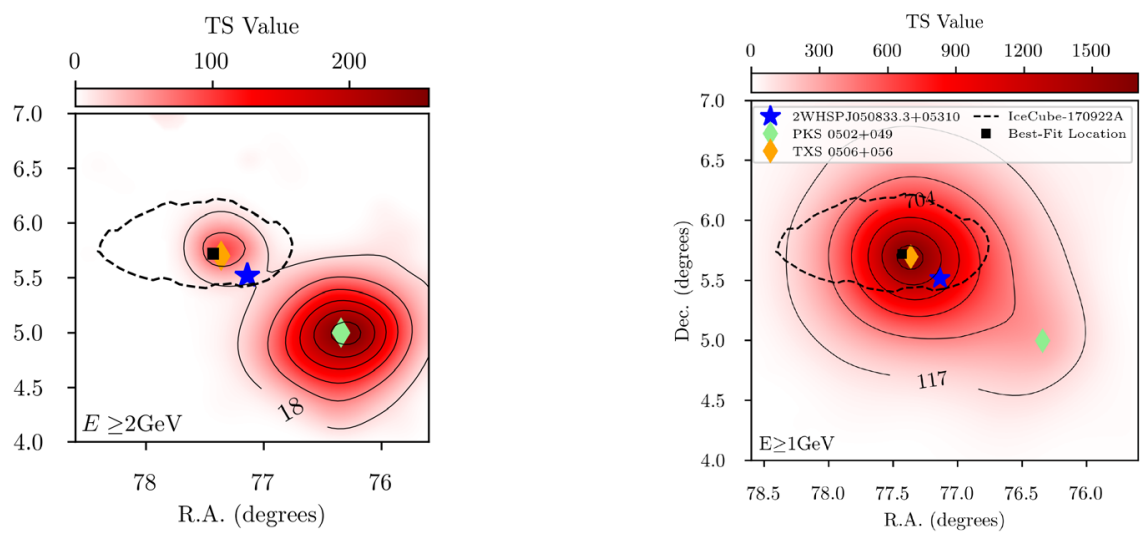

Figure 2. Left: The test-statistic map of the region during the neutrino flare in 2014/2015. PKS $0502+049$ is the dominant $\gamma$-ray emitter in the sky. Above $2 \mathrm{GeV}$ the sources are disentangled and above $5 \mathrm{GeV}$ TXS $0506+056$ is the dominating source. This is due to the log-parabola spectrum of PKS 0502+049 that softens above $934 \mathrm{MeV} \mathrm{[10].} \mathrm{For} \mathrm{symbol} \mathrm{explanation} \mathrm{see} \mathrm{right} \mathrm{plot.} \mathrm{Right:} \mathrm{The}$ TS map between MJD 57908 and 58018, just before the neutrino alert. Here TXS 0506+056 dominates already at $1 \mathrm{GeV}$.

of 2WHSP J050833.3+05310. This result is confirmed by a dedicated analysis at the source's 
location. For the other two source the picture differs between the two time periods around the neutrino flare and the neutrino alert, respectively. During the neutrino flare PKS 0502+049 dominates the sky below several GeV. A dedicated light curve (Figure 3, right) reveals the time evolution of this emission. There are two peaks in the photon flux only before and after
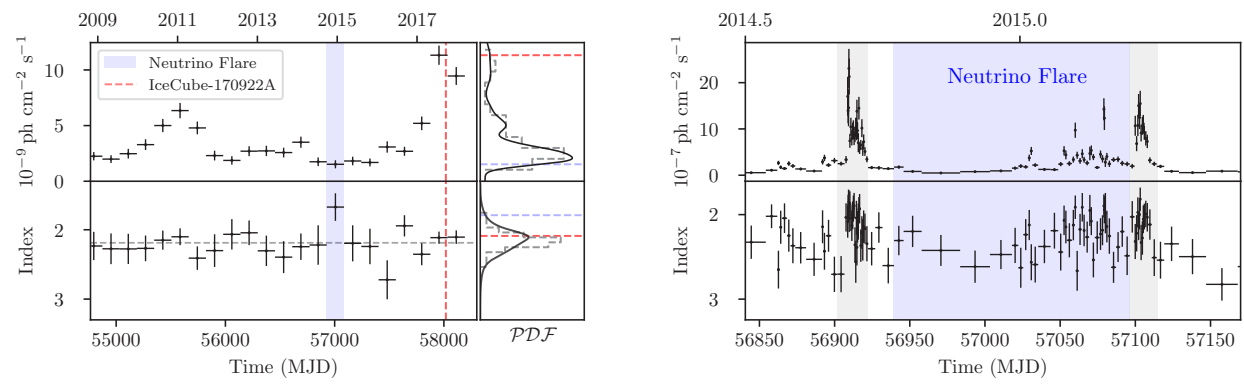

Figure 3. Left: The 158 days, (fixed-binning) $\gamma$-ray light curve of TXS $0506+056$ above $2 \mathrm{GeV}$. The kernel density estimate of the flux and spectral index distribution are shown as black lines on the plots on the top- and bottom-right, respectively. Right: An adaptive bin light curve for PKS 0502+049 above Emin $=214 \mathrm{MeV}$ (see [11] for technical explanation). Note that we go down to lower energies since the PKS $0502+049$ strongly dominates in this energy regime. Going to higher energies it quickly softens above $934 \mathrm{MeV}$ [10]. The grey shaded area shows the time windows of an increased photon flux. In both plots the blue band indicates the time window of the neutrino flare.

the neutrino flare. Assuming a certain level of time synchronized emission this is an argument against a connection of the IceCube neutrinos and the $\gamma$-ray flare of this object. At the same time the $\gamma$-ray flux from TXS $0506+056$ is low, but shows a hard spectrum with photon index of $1.67 \pm 0.2$. The probability of observing such a hard state under the hypothesis of a constant flux is evaluated from a kernel density estimation to $\sim 1 \%$. At the arrival time of IceCube170922A, TXS 0506+056 is clearly the dominating object in the region with a $\gamma$-ray flux around 6 times higher than in the quiescent state. The SEDs of TXS 0506+056 during the two relevant time windows are shown in Figure 4. Additionally to the spectrum above $2 \mathrm{GeV}$ as discussed in [7], we additionally tried to model the source down to $100 \mathrm{MeV}$. The blue band indicates a fit to the data between $100 \mathrm{MeV}$ and $31.6 \mathrm{GeV}$ when using the shape of the best-fit 9.5 year spectrum and only leave the normalisation free. It can be seen that this well reproduces the low-energy part of the SED, while being in tension with the SED above 30 $\mathrm{GeV}$.

\section{Conclusion}

IceCube has published two cases of neutrino emission that are spatially well consistent with emission from the IBL/HBL Blazar TXS 0506+056. Looking into all the available multiwavelength data we provide additional information that supports this picture. While there are other non-thermal sources close by, only one of them, PKS $0502+049$, is also a $\gamma$-ray emitter. The flare of PKS $0502+049$ is shown to be not synchronized with the neutrino emission. Furthermore the $\gamma$-ray spectra of TXS $0506+056$ during both neutrino emission periods are well consistent with the flux level observed in neutrinos. In fact a hardening of the spectrum during the neutrino flare could point to a direct connection of neutrinos and $\gamma$-rays. Although statistically still weak, this provides potentially important insights in the 

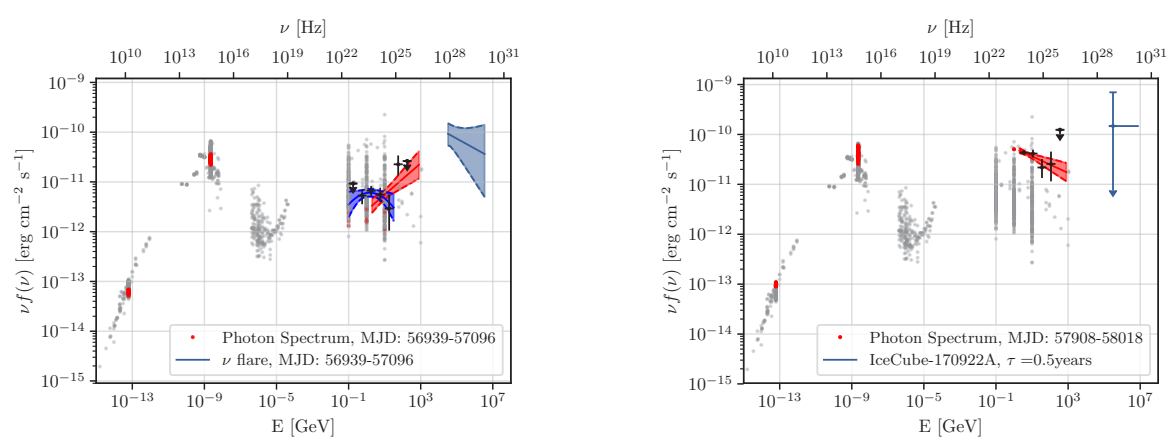

Figure 4. Left: The hybrid SED of electromagnetic emission and neutrinos during the period of the neutrino flare. Archival and time simultaneous data are shown in grey and color, respectively. The red band shows the $\gamma$-ray spectrum for neutrino energies above $2 \mathrm{GeV}$, while the blue band shows a fit to the data between $100 \mathrm{MeV}$ and $30 \mathrm{GeV}$ when fixing the spectrum to the 9.5 year Fermi-LAT best-fit shape and only leaving the normalization free. Right: The same as the left plot around the time of the neutrino alert. As there is only one event observed we can only place an upper limit for the neutrino flux derived from Poisson statistics for half a year of IceCube exposure. In both plots the SED points are EBL de-absorbed using the model of [12].

production mechanisms of neutrinos in blazar jets. This scenario is also discussed in several theoretical work, e.g. [13] [14].

\section{References}

[1] M.G. Aartsen et al. (IceCube), Astrophys. J. 833, 3 (2016), 1607.08006

[2] M.G. Aartsen et al. (IceCube) (2018), 1811.07979

[3] M.G. Aartsen et al. (IceCube), Astropart. Phys. 92, 30 (2017), 1612.06028

[4] S. Paiano, R. Falomo, A. Treves, R. Scarpa, Astrophys. J. 854, L32 (2018), 1802 . 01939

[5] M.G. Aartsen et al. (IceCube, Fermi-LAT, MAGIC, AGILE, ASAS-SN, HAWC, H.E.S.S., INTEGRAL, Kanata, Kiso, Kapteyn, Liverpool Telescope, Subaru, Swift NuSTAR, VERITAS, VLA/17B-403), Science 361, eaat1378 (2018), 1807.08816

[6] M.G. Aartsen et al. (IceCube), Science 361, 147 (2018), 1807.08794

[7] P. Padovani, P. Giommi, E. Resconi, T. Glauch, B. Arsioli, N. Sahakyan, M. Huber, Mon. Not. Roy. Astron. Soc. 480, 192 (2018), 1807.04461

[8] P. Padovani, F. Oikonomou, M. Petropoulou, P. Giommi, E. Resconi (2019), 1901.06998

[9] E. Massaro, A. Maselli, C. Leto, P. Marchegiani, M. Perri, P. Giommi, S. Piranomonte, Astrophys. Space Sci. 357, 75 (2015), 1502.07755

[10] F. Acero et al. (Fermi-LAT), Astrophys. J. Suppl. 218, 23 (2015), 1501.02003

[11] B. Lott, L. Escande, S. Larsson, J. Ballet, 544, A6 (2012), 1201.4851

[12] R.C. Gilmore, R.S. Somerville, J.R. Primack, A. Domínguez, 422, 3189 (2012), 1104.0671

[13] K. Wang, R.Y. Liu, Z. Li, X.Y. Wang, Z.G. Dai (2018), 1809.00601

[14] X. Rodrigues, S. Gao, A. Fedynitch, A. Palladino, W. Winter (2018), 1812.05939 